\title{
PRODUCTS OF SPECIAL SETS OF REAL NUMBERS
}

\author{
BOAZ TSABAN AND TOMASZ WEISS
}

\begin{abstract}
We describe a simple machinery which translates results on algebraic sums of sets of reals into the corresponding results on their cartesian product. Some consequences are:

(1) The product of a meager/null-additive set and a strong measure zero/strongly meager set in the Cantor space has strong measure zero/is strongly meager, respectively.

(2) Using Scheepers' notation for selection principles: $\mathrm{S}_{\text {fin }}\left(\Omega, \Omega^{g p}\right) \cap \mathrm{S}_{1}(\mathcal{O}, \mathcal{O})=$ $\mathrm{S}_{1}\left(\Omega, \Omega^{g p}\right)$, and Borel's Conjecture for $\mathrm{S}_{1}(\Omega, \Omega)$ (or just $\mathrm{S}_{1}\left(\Omega, \Omega^{g p}\right)$ ) implies Borel's Conjecture.

These results extend results of Scheepers and Miller, respectively.
\end{abstract}

\section{Products in the Cantor space}

The Cantor space $\mathcal{C}=\{0,1\}^{\mathbb{N}}$ is equipped with the product topology. For distinct $x, y \in \mathcal{C}$, write $N(x, y)=\min \{n: x(n) \neq y(n)\}$. Then the topology of $\mathcal{C}$ is generated by the following metric:

$$
d(x, y)= \begin{cases}\frac{1}{N(x, y)+1} & x \neq y \\ 0 & x=y\end{cases}
$$

(so that $d(x, y) \leq 1$ for all $x, y \in \mathcal{C}$ ). A canonical measure $\mu$ is defined on $\mathcal{C}$ by taking the product of the uniform probability measure on $\{0,1\}$. Fix a natural number $k$, and consider the product space $\mathcal{C}^{k}$. Define the product metric $d_{k}$ on $\mathcal{C}^{k}$ by

$$
d_{k}\left(\left(x_{0}, \ldots, x_{k-1}\right),\left(y_{0}, \ldots, y_{k-1}\right)\right)=\max \left\{d\left(x_{0}, y_{0}\right), \ldots, d\left(x_{k-1}, y_{k-1}\right)\right\} .
$$

Then $d_{k}$ generates the topology of $\mathcal{C}^{k}$. The measure on $\mathcal{C}^{k}$ is the product measure $\mu \times \ldots \times \mu$ ( $k$ times).

$\mathcal{C}$, with the operation $\oplus$ defined by $(x \oplus y)(n)=x(n)+y(n) \bmod 2$ is a topological group, and therefore so is $\mathcal{C}^{k}$ for all $k$.

Lemma 1.1. The function $\Psi_{k}: \mathcal{C}^{k} \rightarrow \mathcal{C}$ defined by

$$
\Psi_{k}\left(x_{0}, \ldots, x_{k-1}\right)(m k+i)=x_{i}(m)
$$

for each $m$ and each $i<k$, is a bi-Lipschitz measure preserving group isomorphism.

1991 Mathematics Subject Classification. Primary: 26A03; Secondary: 37F20, 03E75.

Key words and phrases. Special sets of real numbers, products. 
Proof. Clearly $\Psi_{k}$ is bijective. Assume that $\vec{x}=\left(x_{0}, \ldots, x_{k-1}\right)$ and $\vec{y}=\left(y_{0}, \ldots, y_{k-1}\right)$ are members of $\mathcal{C}^{k}$. Then for each $m$ and each $i<k$,

$$
\begin{aligned}
& \Psi_{k}(\vec{x} \oplus \vec{y})(m k+i)= \\
& \quad=\Psi_{k}\left(x_{0} \oplus y_{0}, \ldots, x_{k-1} \oplus y_{k-1}\right)(m k+i)=\left(x_{i} \oplus y_{i}\right)(m)= \\
& \quad=x_{i}(m)+y_{i}(m) \bmod 2=\Psi_{k}(\vec{x})(m k+i)+\Psi_{k}(\vec{y})(m k+i) \bmod 2 .
\end{aligned}
$$

Thus, $\Psi_{k}(\vec{x} \oplus \vec{y})=\Psi_{k}(\vec{x}) \oplus \Psi_{k}(\vec{y})$, and $\Psi_{k}$ is a group isomorphism.

Now, assume that $\vec{x}=\left(x_{0}, \ldots, x_{k-1}\right)$ and $\vec{y}=\left(y_{0}, \ldots, y_{k-1}\right)$ are distinct members of $\mathcal{C}^{k}$, and let $i$ be such that $d\left(x_{i}, y_{i}\right)$ is maximal, that is, $N=N\left(x_{i}, y_{i}\right)$ is minimal. Then $N\left(\Psi_{k}(\vec{x}), \Psi_{k}(\vec{y})\right) \geq k N$, and therefore

$$
d\left(\Psi_{k}(\vec{x}), \Psi_{k}(\vec{y})\right) \leq \frac{1}{k N+1}<d(x, y) .
$$

Similarly, for distinct $x, y \in \mathcal{C}$, if $N(x, y)=m k+i$ where $i<k$, then $N\left(\Psi_{k}^{-1}(x)\right.$, $\left.\Psi_{k}^{-1}(y)\right) \geq m$ and

$$
d\left(\Psi_{k}^{-1}(x), \Psi_{k}^{-1}(y)\right) \leq \frac{1}{m+1} \leq \frac{k}{m k+i+1}=k \cdot d(x, y) .
$$

To see that $\Psi_{k}$ is measure preserving, observe that the measure of a basic open set $U$ in $\mathcal{C}$ is $2^{-m}$, where $m$ is the number of coordinates of $U$ which are not equal to $\{0,1\}$. Consequently, the same assertion is true for $\mathcal{C}^{k}$, where $m$ is the sum of numbers of such coordinates within each of the $k$ coordinates of $\mathcal{C}^{k}$. This number $m$ is invariant under $\Psi_{k}$; thus $\Psi_{k}$ preserves measures of basic open sets.

This often allows us to restrict attention to subsets of $\mathcal{C}$ rather than subsets of $\mathcal{C}^{k}$ for arbitrary $k$.

Abbreviation 1.2. $\tilde{P}(A)=\bigcup_{k \in \mathbb{N}} P\left(A^{k}\right)$.

Theorem 1.3. Assume that $\mathcal{I}, \mathcal{J}, \mathcal{K} \subseteq \tilde{P}(\mathcal{C})$, and that $\mathcal{K}$ is closed under taking Lipschitz images. If

$$
\text { for each } X \in \mathcal{I} \cap P\left(\mathcal{C}^{k}\right) \text { and } Y \in \mathcal{J} \cap P\left(\mathcal{C}^{k}\right), X \times Y \in \mathcal{K} \text {, }
$$

then

for each $X \in \mathcal{I} \cap P\left(\mathcal{C}^{k}\right)$ and $Y \in \mathcal{J} \cap P\left(\mathcal{C}^{k}\right), X \oplus Y \in \mathcal{K}$.

Proof. The mapping $(x, y) \mapsto x \oplus y$ is a Lipschitz mapping from $X \times Y$ onto $X \oplus Y$.

The converse of Theorem 1.3 also holds, and in a much stronger form. For simplicity, we introduce the following notions.

Abbreviation 1.4. $\mathcal{P} \subseteq \tilde{P}(\mathcal{C})$ is semiproductive if:

(1) For each $k, l$, and $X \in \mathcal{P} \cap \mathcal{C}^{k}$, if 0 is the zero element of $\mathcal{C}^{l}$, then $X \times\{0\} \in \mathcal{P}$; and

(2) For each $k, l, X \in \mathcal{P} \cap \mathcal{C}^{k}$, and a bi-Lipschitz measure preserving group isomorphism $\Phi: \mathcal{C}^{k} \rightarrow \mathcal{C}^{l}, \Phi[X] \in \mathcal{P}$.

We will say that $\mathcal{P}$ is 0 -productive if we only require that (1) is satisfied, and isoproductive if we only require that (2) is satisfied. 
Many properties of special sets of reals are semiproductive, e.g., Hausdorff dimension, strong measure zero, the properties in Cichoń's Diagram for small sets [8] or in Scheepers' Diagram (see Section 4) and its extensions $[12,13]$; see [5, 10] for more examples.

As changing the order of coordinates is a bi-Lipschitz measure preserving group isomorphism, we have the following.

Lemma 1.5. Assume that $\mathcal{P} \subseteq \tilde{P}(\mathcal{C})$ is semiproductive. Then for each $k, l$, and $X \in \mathcal{P} \cap \mathcal{C}^{k}$, if 0 is the zero element of $\mathcal{C}^{l}$, then $\{0\} \times X \in \mathcal{P}$.

Theorem 1.6. Assume that $\mathcal{I}, \mathcal{J}, \mathcal{K} \subseteq \tilde{P}(\mathcal{C})$ such that $\mathcal{I}$ and $\mathcal{J}$ are semiproductive, and $\mathcal{K}$ is iso-productive. If

for each $X \in \mathcal{I} \cap P(\mathcal{C})$ and $Y \in \mathcal{J} \cap P(\mathcal{C}), X \oplus Y \in \mathcal{K}$,

then

for each $X \in \mathcal{I}$ and $Y \in \mathcal{J}, X \times Y \in \mathcal{K}$.

Proof. Assume that $X \in \mathcal{I} \cap P\left(\mathcal{C}^{k}\right)$ and $Y \in \mathcal{J} \cap P\left(\mathcal{C}^{l}\right)$. Then $\tilde{X}=\Psi_{k}[X] \in \mathcal{I} \cap P(\mathcal{C})$, and $\tilde{Y}=\Psi_{l}[Y] \in \mathcal{J} \cap P(\mathcal{C})$.

$$
\begin{aligned}
\Psi_{2}[\tilde{X} \times \tilde{Y}] & =\Psi_{2}[(\tilde{X} \times\{0\}) \oplus(\{0\} \times \tilde{Y})]= \\
& =\Psi_{2}[\tilde{X} \times\{0\}] \oplus \Psi_{2}[\{0\} \times \tilde{Y}]=X^{\prime} \oplus Y^{\prime},
\end{aligned}
$$

Thus, $X^{\prime} \oplus Y^{\prime} \in \mathcal{K}$. As $\Psi_{2}$ is bijective, $\tilde{X} \times \tilde{Y}=\Psi_{2}^{-1}\left[X^{\prime} \oplus Y^{\prime}\right] \in \mathcal{K}$. As $\Psi_{k} \times \Psi_{l}$ is a bi-Lipschitz measure preserving group isomorphism and $\Psi_{k}^{-1} \times \Psi_{l}^{-1}: \tilde{X} \times \tilde{Y} \rightarrow X \times Y$ is surjective, $X \times Y \in \mathcal{K}$.

Assume that $\mathcal{I} \subseteq \tilde{P}(\mathcal{C})$. A subset $X$ of $\mathcal{C}^{k}$ is $\mathcal{I}$-additive if for each $I \in \mathcal{I} \cap P\left(\mathcal{C}^{k}\right)$, $X \oplus I \in \mathcal{I}$. Clearly if $X, Y \subseteq \mathcal{C}$ are $\mathcal{I}$-additive, then $X \oplus Y$ is $\mathcal{I}$-additive. More generally, a subset $X$ of $\mathcal{C}^{k}$ is $(\mathcal{I}, \mathcal{J})$-additive if for each $I \in \mathcal{I} \cap \mathcal{C}^{k}, X \oplus I \in \mathcal{J}$. Let $\mathcal{I}^{\star}$ and $(\mathcal{I}, \mathcal{J})^{\star}$ denote the classes of all $\mathcal{I}$-additive sets and $(\mathcal{I}, \mathcal{J})$-additive sets, respectively.

Theorem 1.7. Assume that $\mathcal{I}, \mathcal{J}, \mathcal{K} \subseteq \tilde{P}(\mathcal{C})$ are iso-productive, and $(\mathcal{J}, \mathcal{K})^{\star},(\mathcal{I}, \mathcal{J})^{\star}$ are 0 -productive. Then for each $X \in(\mathcal{J}, \mathcal{K})^{\star}$ and $Y \in(\mathcal{I}, \mathcal{J})^{\star}, X \times Y \in(\mathcal{I}, \mathcal{K})^{\star}$. In particular, if $\mathcal{I}$ is iso-productive and $\mathcal{I}^{\star}$ is 0 -productive, then $\mathcal{I}^{\star}$ is closed under taking finite products.

Proof. Assume that $X \in(\mathcal{J}, \mathcal{K})^{\star} \cap P(\mathcal{C}), Y \in(\mathcal{I}, \mathcal{J})^{\star} \cap P(\mathcal{C})$, and $I \in \mathcal{I}$. Then $Y \oplus I \in \mathcal{J}$ and therefore $X \oplus(Y \oplus I) \in \mathcal{K}$. Thus $X \oplus Y \in(\mathcal{I}, \mathcal{K})^{\star}$.

Lemma 1.8. Assume that $\mathcal{I}, \mathcal{J} \subseteq \tilde{P}(\mathcal{C})$ are iso-productive. Then $(\mathcal{I}, \mathcal{J})^{\star}$ is isoproductive.

Proof. Assume that $X \in(\mathcal{I}, \mathcal{J})^{\star} \cap P\left(\mathcal{C}^{k}\right)$ and $\Phi: \mathcal{C}^{k} \rightarrow \mathcal{C}^{l}$ is a bi-Lipschitz measure preserving group isomorphism. Then for each $I \in \mathcal{I} \cap P\left(\mathcal{C}^{l}\right)$,

$$
\Phi[X] \oplus I=\Phi\left[X \oplus \Phi^{-1}[I]\right] .
$$

As $\Phi^{-1}[I] \in \mathcal{I}, X \oplus \Phi^{-1}[I] \in \mathcal{J}$ and therefore $\Phi[X] \oplus I \in \mathcal{J}$, that is, $\Phi[X] \in$ $(\mathcal{I}, \mathcal{J})^{\star}$. 
Thus $(\mathcal{J}, \mathcal{K})^{\star}$ and $(\mathcal{I}, \mathcal{J})^{\star}$ are semiproductive, and the theorem follows from Theorem 1.6.

Following is a useful criterion for the 0-productivity required in Theorem 1.7.

Lemma 1.9. Assume that $\mathcal{I}, \mathcal{J} \subseteq \tilde{P}(\mathcal{C})$ are iso-productive, and for the element $0 \in \mathcal{C}$ and each $X \in(\mathcal{I}, \mathcal{J})^{\star} \cap P(\mathcal{C}), \Psi_{2}[X \times\{0\}] \in(\mathcal{I}, \mathcal{J})^{\star}$. Then $(\mathcal{I}, \mathcal{J})^{\star}$ is 0-productive. In particular, if $\mathcal{I}$ is iso-productive and and for each $X \in \mathcal{I}^{\star} \cap P(\mathcal{C}), \Psi_{2}[X \times\{0\}] \in \mathcal{I}^{\star}$, then $\mathcal{I}^{\star}$ is 0 -productive.

Proof. Assume that $X \in(\mathcal{I}, \mathcal{J})^{\star} \cap P\left(\mathcal{C}^{k}\right)$ and fix $l$. By Lemma 1.8, $\tilde{X}=\Psi_{k}[X] \in$ $(\mathcal{I}, \mathcal{J})^{\star} \cap P(\mathcal{C})$. Thus $\Psi_{2} \circ\left(\Psi_{k} \times \Psi_{l}\right)[X \times\{0\}]=\Psi_{2}[\tilde{X} \times\{0\}] \in(\mathcal{I}, \mathcal{J})^{\star}$. Applying Lemma 1.8 again, we get that $X \times\{0\} \in(\mathcal{I}, \mathcal{J})^{\star}$.

We now give some applications. Let $X$ be a metric space. Following Borel, we say that $X$ has strong measure zero if for each sequence of positive reals $\left\{\epsilon_{n}\right\}_{n \in \mathbb{N}}$, there exists a cover $\left\{I_{n}\right\}_{n \in \mathbb{N}}$ of $X$ such that $\operatorname{diam}\left(I_{n}\right)<\epsilon_{n}$ for all $n$. $X$ has the Hurewicz property if for each sequence $\left\{\mathcal{U}_{n}\right\}_{n \in \mathbb{N}}$ of open covers of $X$ there exist finite subsets $\mathcal{F}_{n} \subseteq \mathcal{U}_{n}, n \in \mathbb{N}$, such that $X \subseteq \bigcup_{n} \bigcap_{m>n} \cup \mathcal{F}_{n}$. Let SMZ (respectively, $\mathcal{H}$ ) denote the collections of metric spaces having strong measure zero (respectively, the Hurewicz property).

The following theorem of Scheepers will serve as a "test case" for our approach.

Theorem 1.10 (Scheepers [11]). Let $X$ be a strong measure zero metric space which also has the Hurewicz property. Then for each strong measure zero metric space $Y$, $X \times Y$ has strong measure zero.

Scheepers' proof of Theorem 1.10 is by a reduction of the Hurewicz property to the so called "grouping property", which is proved using a result from topological game-theory. We will present alternative proofs for the case that the spaces are sets of real numbers. We first observe that this follows from the corresponding theorem with $X \oplus Y$ instead of $X \times Y$, which was proved in [7]: Since the collections $\mathcal{H} \cap \tilde{P}(\mathcal{C})$ and SMZ $\cap \tilde{P}(\mathcal{C})$ are semiproductive, Theorem 1.6 applies. (For another proof of Scheepers' Theorem in $\mathcal{C}$, see Theorem A.2 in the appendix.)

We now treat the classes of meager-additive and null-additive sets. Let $\mathcal{M}$ and $\mathcal{N}$ denote the meager (i.e., first category) and null (i.e., measure zero) sets, respectively.

Theorem 1.11. $\mathcal{M}^{\star} \cap \tilde{P}(\mathcal{C})$ and $\mathcal{N}^{\star} \cap \tilde{P}(\mathcal{C})$ are semiproductive, and are closed under taking finite products.

Proof. Clearly, $\mathcal{M} \cap \tilde{P}(\mathcal{C})$ and $\mathcal{N} \cap \tilde{P}(\mathcal{C})$ are semiproductive. By Theorem 1.7, it is enough to show that the classes $\mathcal{M}^{\star} \cap \tilde{P}(\mathcal{C})$ and $\mathcal{N}^{\star} \cap \tilde{P}(\mathcal{C})$ are 0-productive. We first treat $\mathcal{M}^{\star}$.

Lemma 1.12 (Bartoszyński-Judah [1, Theorem 2.7.17]). A subset $X$ of $\mathcal{C}$ is meageradditive if, and only if, for each increasing sequence $\left\{m_{n}\right\}_{n \in \mathbb{N}}$ there exist a sequence $\left\{l_{n}\right\}_{n \in \mathbb{N}}$ and $y \in \mathcal{C}$ such that for each $x \in X$ and all but finitely many $n$,

$$
l_{n} \leq m_{k}<m_{k+1} \leq l_{n+1} \text { and } x \uparrow\left[m_{k}, m_{k+1}\right)=y \uparrow\left[m_{k}, m_{k+1}\right)
$$


for some $k$. (In this case we say that $\left\{l_{n}\right\}_{n \in \mathbb{N}}$ and $y$ are appropriate for $\left\{m_{n}\right\}_{n \in \mathbb{N}}$ and $X$.

We will prove the sufficient criterion of Lemma 1.9. Assume that $X \in \mathcal{M}^{\star} \cap P(\mathcal{C})$, and let $\tilde{X}=\Psi_{2}[X \times\{0\}]$. We must show that $\tilde{X} \in \mathcal{M}^{\star}$. Let an increasing sequence $\left\{m_{n}\right\}_{n \in \mathbb{N}}$ be given. Choose an increasing sequence $\left\{m_{n}^{\prime}\right\}_{n \in \mathbb{N}}$ of even numbers such that for all but finitely many $n$, there exists $k$ such that $m_{n}^{\prime} \leq m_{k}<m_{k+1} \leq m_{n+1}^{\prime}$.

Apply Lemma 1.12 to obtain $\left\{l_{n}\right\}_{n \in \mathbb{N}}$ and $y$ which are appropriate for $\left\{m_{n}^{\prime} / 2\right\}_{n \in \mathbb{N}}$ and $X$. By the definition of $\Psi_{2}$, we get that $\left\{2 l_{n}\right\}_{n \in \mathbb{N}}$ and $\Psi_{2}(y, 0)$ are appropriate for $\left\{m_{n}^{\prime}\right\}_{n \in \mathbb{N}}$ and $\Psi_{2}[X \times\{0\}]$. In particular, they are appropriate for $\left\{m_{n}\right\}_{n \in \mathbb{N}}$ and $\Psi_{2}[X \times\{0\}]$. This shows that $\mathcal{M}^{\star}$ is 0 -productive.

Using similar arguments, the fact that $\mathcal{N}^{\star} \cap \tilde{P}(\mathcal{C})$ is 0 -productive follows from the following.

Lemma 1.13 (Shelah [1, Theorem 2.7.18]). A subset $X$ of $\mathcal{C}$ is null-additive if, and only if, for every increasing sequence $\left\{m_{n}\right\}_{n \in \mathbb{N}}$ there exists a sequence $\left\{S_{n}\right\}_{n \in \mathbb{N}}$ such that each $S_{n}$ is a set of at most $n$ functions from $\left[m_{n}, m_{n+1}\right)$ to $\{0,1\}$, and for each $x \in X$ and all but finitely many $n, x \uparrow\left[m_{n}, m_{n+1}\right) \in S_{n}$.

This finishes the proof of Theorem 1.11.

Proposition 1.14 (folklore). For all $k$, a set $X \subseteq \mathcal{C}^{k}$ has strong measure zero if, and only if, for each meager $M \subseteq \mathcal{C}^{k}, X \oplus M \neq \mathcal{C}^{k}$.

Proof. Assume that $X \subseteq \mathcal{C}^{k}$ has strong measure, and $M \subseteq \mathcal{C}^{k}$ is meager. Then by the Galvin-Mycielski-Solovay Theorem, $\Psi_{k}[X \oplus M]=\Psi_{k}[X] \oplus \Psi_{k}[M] \neq \mathcal{C}$, and therefore $X \oplus M \neq \mathcal{C}^{k}$.

Conversely, assume that $X \subseteq \mathcal{C}^{k}$ and for each meager $M \subseteq \mathcal{C}^{k}, X \oplus M \neq \mathcal{C}^{k}$. Then for each meager $M \subseteq \mathcal{C}, X \oplus \Psi_{k}^{-1}[M] \neq \mathcal{C}^{k}$, therefore $\Psi_{k}\left[X \oplus \Psi_{k}^{-1}[M]\right]=\Psi_{k}[X] \oplus M \neq$ $\mathcal{C}$, thus $\Psi_{k}[X]$ has strong measure zero, and therefore $X$ has strong measure zero.

Every set of reals with the Hurewicz property as well as strong measure zero is meager-additive ([7], or Theorem A.3 below). Consequently, the following theorem extends Scheepers' Theorem 1.10 in the case that $X, Y \subseteq \mathcal{C}$.

Theorem 1.15. Assume that $X \in \mathcal{M}^{\star} \cap \tilde{P}(\mathcal{C})$ and $Y \in \operatorname{SMZ} \cap \tilde{P}(\mathcal{C})$. Then $X \times Y \in$ SMZ.

Proof. By Theorem 1.11, $\mathcal{M}^{\star} \cap \tilde{P}(\mathcal{C})$ is semiproductive. Recall that $\operatorname{SMZ} \cap \tilde{P}(\mathcal{C})$ is semiproductive too. By the Galvin-Mycielski-Solovay Theorem (Proposition 1.14 for $k=1$ ), the conditions of Theorem 1.6 hold, and its consequence tells what we are looking for.

To prove the dual result, we need the following lemma. For a set $J$ denote $J_{x}=$ $\{y:(x, y) \in J\}$ and $J^{y}=\{x:(x, y) \in J\}$. Say that a family $\mathcal{J}$ which does not contain any $\mathcal{C}^{k}$ as element is a Fubini family if, whenever $J \in \mathcal{J} \cap \mathcal{C}^{k+l}$, we have that

$$
\left\{x \in \mathcal{C}^{k}: J_{x} \notin \mathcal{J}\right\} \in \mathcal{J} \text {, and }\left\{y \in \mathcal{C}^{l}: J^{y} \notin \mathcal{J}\right\} \in \mathcal{J} \text {. }
$$


The most important examples for Fubini families are $\mathcal{M}$ (Kuratowski-Ulam Theorem) and $\mathcal{N}$ (Fubini Theorem). To understand what we really prove, we will say that $\mathcal{J}$ is a weakly Fubini family if " $\in \mathcal{J}$ " and " $\in \mathcal{J}$ " in (1) are replaced by " $\neq \mathcal{C}^{k}$ " and $" \neq \mathcal{C}^{l} "$, respectively. Clearly, each Fubini family is a weakly Fubini family.

A set $X \subseteq \mathcal{C}^{k}$ is not $\mathcal{J}$-covering if for each $J \in \mathcal{J} \cap P\left(\mathcal{C}^{k}\right), X \oplus J \neq \mathcal{C}^{k}$.

Lemma 1.16. Assume that $\mathcal{J}$ is a weakly Fubini family. Then the family of not $\mathcal{J}$-covering sets is 0 -productive.

Proof. Assume that $X \subseteq \mathcal{C}^{k}$ is not $\mathcal{J}$-covering, $0 \in \mathcal{C}^{l}$, and $J \in \mathcal{J} \cap P\left(\mathcal{C}^{k+l}\right)$. As $\mathcal{J}$ is a weakly Fubini family, there exists $y \in \mathcal{C}^{l}$ such that $J^{y} \in \mathcal{J}$. Thus, $((X \times\{0\}) \oplus J)^{y}=$ $X \oplus J^{y} \neq \mathcal{C}^{k}$, therefore $(X \times\{0\}) \oplus J \neq \mathcal{C}^{k+l}$.

A set $X \subseteq \mathcal{C}^{k}$ is strongly meager if it is not $\mathcal{N}$-covering. Using the same proof as in Theorem 1.15, we get the following.

Theorem 1.17. The product of a null-additive set in $\mathcal{C}^{k}$ and a strongly meager set in $\mathcal{C}^{l}$ is strongly meager.

It is folklore that a product of strong measure zero sets need not have strong measure zero (e.g., [5]), and that the product of strongly meager sets need not be strongly meager. To see the last assertion, we say that a set $S \subseteq \mathcal{C}$ is $\kappa$-Sierpinski if $|S| \geq \kappa$ but for each null set $N,|S \cap N|<\kappa$. Observe that the diagonal is null in $\mathcal{C}^{2}$.

Theorem 1.18. Assume that $\operatorname{cov}(\mathcal{N})=\mathfrak{b}=\mathfrak{c}$. Then there exists a strongly meager set of reals $S \subseteq \mathcal{C}$ such that $S^{2} \oplus \Delta=\mathcal{C}^{2}$, where $\Delta=\{(x, x): x \in \mathcal{C}\}$.

Proof. Since $\operatorname{cov}(\mathcal{N})=\mathfrak{c}$ we can construct, as in [12, Lemma 42], a $\operatorname{cov}(\mathcal{N})$-Sierpiński set $S$ such that $S \oplus S=\mathcal{C}$. Then $S^{2} \oplus \Delta=\mathcal{C}^{2}$ : Given $y, z \in \mathcal{C}$, choose $s, t \in S$ such that $s \oplus t=y \oplus z$, and take $x=s \oplus y$. Then $s \oplus x=y$, and

$$
t \oplus x=t \oplus(s \oplus s) \oplus x=(s \oplus t) \oplus(s \oplus x)=(y \oplus z) \oplus y=z,
$$

thus $(s, t) \oplus(x, x)=(y, z)$. Since $\mathfrak{b}=\operatorname{cov}(\mathcal{N}), S$ is a $\mathfrak{b}$-Sierpiński set and by $[12$, p. 376], every Borel image of $S$ is bounded. Moreover, for each null set $N,|S \cap N|<$ $\operatorname{cov}(\mathcal{N})$. By a result of Pawlikowski (see [1] - Definition 8.5.7, the observation after it, and Theorem 8.5.12), these two properties imply that $S$ is strongly meager.

\section{Products in the EuClidean space}

As the mapping from $\mathbb{R}^{k} \times \mathbb{R}^{k}$ to $\mathbb{R}^{k}$ defined by $(x, y) \mapsto x+y$ is Lipschitz, Theorem 1.3 remains true in the Euclidean space $\mathbb{R}^{k}$. However, we are unable to prove Theorem 1.6 (in its current form) for the Euclidean space $\left(\left\langle\mathbb{R}^{k},+\right\rangle\right.$ and $\langle\mathbb{R},+\rangle$ are not homeomorphic: $\mathbb{R}^{k}$ remains connected after removing a point). We can, though, obtain similar results.

Abbreviation 2.1. A collection $\mathcal{P} \subseteq \tilde{P}(\mathbb{R})$ is bi-0-productive if for each $k, l$, and $X \in \mathcal{P} \cap \mathbb{R}^{k}$, if 0 is the zero element of $\mathbb{R}^{l}$, then $X \times\{0\},\{0\} \times X \in \mathcal{P}$.

Theorem 2.2. Assume that $\mathcal{I}, \mathcal{J}, \mathcal{K} \subseteq \tilde{P}(\mathbb{R})$ and $\mathcal{I}, \mathcal{J}$ are bi-0-productive. If 
for each $k$ and all $X \in \mathcal{I} \cap P\left(\mathbb{R}^{k}\right)$ and $Y \in \mathcal{J} \cap P\left(\mathbb{R}^{k}\right), X+Y \in \mathcal{K}$,

then

for each $X \in \mathcal{I}$ and $Y \in \mathcal{J}, X \times Y, Y \times X \in \mathcal{K}$.

Proof. Assume that $X \in \mathcal{I} \cap P\left(\mathbb{R}^{k}\right)$ and $Y \in \mathcal{J} \cap P\left(\mathbb{R}^{l}\right)$. Then

$$
X \times Y=(X \times\{0\})+(\{0\} \times Y) .
$$

As $\mathcal{I}$ and $\mathcal{J}$ are bi-0-productive, $X \times\{0\} \in \mathcal{I} \cap P\left(\mathbb{R}^{k+l}\right)$ and $\{0\} \times Y \in \mathcal{J} \cap P\left(\mathbb{R}^{k+l}\right)$, therefore $X \times Y \in \mathcal{K}$. Similarly, $Y \times X \in \mathcal{K}$.

Theorem 2.3. Assume that $\mathcal{I}, \mathcal{J}, \mathcal{K} \subseteq \tilde{P}(\mathbb{R})$, and that $(\mathcal{J}, \mathcal{K})^{\star}$ and $(\mathcal{I}, \mathcal{J})^{\star}$ are bi-0productive. Then for each $X \in(\mathcal{J}, \mathcal{K})^{\star}$ and $Y \in(\mathcal{I}, \mathcal{J})^{\star}, X \times Y, Y \times X \in(\mathcal{I}, \mathcal{K})^{\star}$. In particular, if $\mathcal{I}^{\star}$ is bi-0-productive, then $\mathcal{I}^{\star}$ is closed under taking finite products.

Proof. Assume that $X \in(\mathcal{J}, \mathcal{K})^{\star} \cap P\left(\mathbb{R}^{k}\right), Y \in(\mathcal{I}, \mathcal{J})^{\star} \cap P\left(\mathbb{R}^{k}\right)$, and $I \in \mathcal{I} \cap P\left(\mathbb{R}^{k}\right)$. Then $Y+I \in \mathcal{J}$ and therefore $X+(Y+I) \in \mathcal{K}$. Thus $X+Y \in(\mathcal{I}, \mathcal{K})^{\star}$. As $(\mathcal{J}, \mathcal{K})^{\star}$ and $(\mathcal{I}, \mathcal{J})^{\star}$ are bi-0-productive, our theorem follows from Theorem 2.2.

It was noticed by Marcin Kysiak that one can use our arguments to obtain Scheepers' Theorem 1.10 in the Euclidean space. To see this, one simply has to generalize the corresponding theorem on sums [7] from $k=1$ to arbitrary $k$. The generalization is straightforward. In Section 3 we show that in fact, this generalization is not necessary.

The analogue of Theorem 1.11 in the Euclidean space does not seem to follow from the results in this paper.

Problem 2.4. Assume that $X \subseteq \mathbb{R}$ is meager- (respectively, null-) additive. Does it follow that $X \times\{0\}$ is meager- (respectively, null-) additive?

Remark 2.5. We can prove that every meager-additive subset of the Cantor space, when viewed as a subset of $\mathbb{R}$, is meager-additive (with respect to the usual addition in $\mathbb{R}$ ); and similarly for null-additive (in both cases, the other direction is still open). Consequently, the classes of meager-additive and null-additive each contains a nontrivial subclass which is preserved under taking finite products.

\section{The Euclidean spaCe through the LOOKING GLASS}

The results in Section 2 are not easy to use, as one should verify first that the additive results given in the literature for $\mathbb{R}$ actually hold in $\mathbb{R}^{k}$ for all $k$. We suggest here another approach, which covers some of the cases of interest.

Definition 3.1. The function $T:\{0,1\}^{\mathbb{N}} \rightarrow[0,1]$ is defined by

$$
x \mapsto \sum_{i \in \mathbb{N}} \frac{x(i)}{2^{i+1}}=0 . x(0) x(1) x(2) \ldots,
$$

where the last term is in base 2 . Let $C$ denote the collection of all eventually constant elements of $\{0,1\}^{\mathbb{N}}$, and $\mathbb{Q}_{2}=T[C]$ denote the 2 -adic rational numbers in $[0,1]$. 
Lemma 3.2 (folklore).

(1) $T$ is a uniformly continuous surjection.

(2) $C$ is countable.

(3) $T:\{0,1\}^{\mathbb{N}} \backslash C \rightarrow[0,1] \backslash \mathbb{Q}_{2}$ is a homeomorphism which preserves measure in both directions.

Proof. (1) $T$ is continuous on its compact domain, and clearly it is onto.

(2) is obvious, and the only nontrivial part of (3) is that $T^{-1}$ is continuous on $[0,1] \backslash \mathbb{Q}_{2}$ (it is not uniformly continuous: Take $x_{n}=0.10^{n} \overline{01}$ and $y_{n}=0.01^{n} \overline{01}$, then $x_{n}-y_{n} \rightarrow 0$, but $d\left(T^{-1}\left(x_{n}\right), T^{-1}\left(y_{n}\right)\right)=1$ for all $n$.). Write $\tilde{y}$ for $T^{-1}(y)$. If $x_{n} \rightarrow x$ are elements of $[0,1] \backslash \mathbb{Q}_{2}$ then from some $n$ onwards, the $\tilde{x}_{n}(0)=\tilde{x}(0)$ : Assume that this is not the case. Then by moving to a subsequence we may assume that for all $n, \tilde{x}(0) \neq \tilde{x}_{n}(0)$. Assume that $\tilde{x}(0)=0$ and $\tilde{x}_{n}(0)=1$ for all $n$ (the other case is similar). Let $k=\min \{m>0: x(m)=0\}$ (recall that $\tilde{x}$ is not eventually constant). Then $x=0.01^{k-1} 0 \ldots$, thus

$$
x_{n}-x \geq 0.1-0.01^{k-1} 0 \overline{1}=0.1-0.01^{k}=0.0^{k} 1=\frac{1}{2^{k+1}}
$$

for all $n$, a contradiction.

An inductive argument shows that for each $k, \tilde{x}_{n}(k)=\tilde{x}(k)$ for all large enough $n$.

In principle, we can use the function $T$ to translate questions about products in $\mathbb{R}$ into questions about products in $\mathcal{C}$, apply the results of Section 1 , and translate back to $\mathbb{R}$. The problem is that in our test-case, Scheepers' Theorem 1.10 in $\mathbb{R}$, we must deal with strong measure zero sets. By (1) of Lemma 3.2, if $Y$ has strong measure zero then so does $T[Y]$. The other direction does not follow from Lemma 3.2, since a homeomorphic image of a strong measure zero set need not have strong measure zero [9] (see [15] for a simple proof).

Proposition 3.3. For each $k, T^{k}: \mathcal{C}^{k} \rightarrow[0,1]^{k}$ preserves strong measure zero sets in both directions.

Proof. Since $T$ is uniformly continuous, $T^{k}$ is uniformly continuous. Thus, if $X \subseteq \mathcal{C}^{k}$ has strong measure zero, then so does $T^{k}[X]$.

We now prove the other direction. The following is an easy exercise.

Lemma 3.4 (folklore). Assume that there exists $f: \mathbb{N} \rightarrow \mathbb{N}$ such that the metric space $(X, d)$ has the following property: For each sequence $\left\{\epsilon_{n}\right\}_{n \in \mathbb{N}}$ of positive reals, there exist a cover $\left\{I_{m}^{n}: n \in \mathbb{N}, m \leq f(n)\right\}$ of $X$ satisfying $\operatorname{diam}\left(I_{m}^{n}\right)<\epsilon_{n}$ for each $n$ and $m$. Then $(X, d)$ has strong measure zero.

To use Lemma 3.4, we make the following observation.

Lemma 3.5. Assume that $I \subseteq[0,1]$ and $\operatorname{diam}(I)<\epsilon$. Then there exist $A_{0}, A_{1} \subseteq \mathcal{C}$, both with diameter $<\epsilon$, such that $T^{-1}[I] \subseteq A_{0} \cup A_{1}$. 
Proof. Let $n$ be the maximal such that $2^{n-1} \epsilon \leq 1$. Then there exists $k<2^{n}-1$ such that

$$
I \subseteq\left[\frac{k}{2^{n}}, \frac{k+2}{2^{n}}\right]=\left[\frac{k}{2^{n}}, \frac{k+1}{2^{n}}\right] \cup\left[\frac{k+1}{2^{n}}, \frac{k+2}{2^{n}}\right] .
$$

There exist sequences $s_{0}, s_{1} \in\{0,1\}^{n}$ such that for $i=0,1$, if $A_{i}=\left\{x \in \mathcal{C}: s_{i} \subseteq x\right\}$, then $T\left[A_{i}\right]=\left[(k+i) / 2^{n},(k+i+1) / 2^{n}\right]$.

Assume that $Y \subseteq[0,1]^{k}$ has strong measure zero, and $\left\{\epsilon_{n}\right\}_{n \in \mathbb{N}}$ is a sequence of positive reals. Let $I_{n} \subseteq[0,1]^{k}$ be such that $\operatorname{diam}\left(I_{n}\right)<\epsilon_{n}$ and $Y \subseteq \bigcup_{n} I_{n}$. Fix $n$. Then $I_{n}$ is contained in the product of $k$ intervals, $I_{1}^{n}, \ldots I_{k}^{n} \subseteq[0,1]$, each with diameter $<\epsilon_{n}$. For each $i=1, \ldots, k$, use Lemma 3.5 to obtain sets $A_{0}^{n, i}, A_{1}^{n, i}$ as in the lemma. Then

$$
I_{n} \subseteq \bigcup_{s \in\{0,1\}^{k}} \Pi_{i=1}^{k} T\left[A_{s(i)}^{n, i}\right]=T^{k}\left[\bigcup_{s \in\{0,1\}^{k}} \Pi_{i=1}^{k} A_{s(i)}^{n, i}\right],
$$

so that $T^{-k}\left[I_{n}\right]$ is covered by $2^{k}$ sets of diameter $<\epsilon_{n}$. Since the sets $I_{n}$ cover $Y$, we have by Lemma 3.4 that $T^{-k}[Y]$ has strong measure zero.

We now show how to prove Scheepers' Theorem in the Euclidean space.

Lemma 3.6. Assume that $\mathcal{I}$ is preserved under taking closed subsets, uniformly continuous images, and countable unions, and that $\mathbb{R} \notin \mathcal{I}$. Then for each $X \in \mathcal{I} \cap P\left(\mathbb{R}^{k}\right)$ and a countable set $Q \subseteq \mathbb{R}$ there exists $x \in \mathbb{R}^{k}$ such that $(x+X) \cap \bigcup_{m<k} \mathbb{R}^{m} \times Q \times$ $\mathbb{R}^{k-m-1}=\emptyset$.

Proof. The assumptions imply that for each $i<k$, the projection $X_{i}=\pi_{i}[X]$ on the $i$ th coordinate is a member of $\mathcal{I}$. As $Q$ is countable, $Q-X_{i} \neq \mathbb{R}$. Choose $x_{i} \notin Q-X_{i}$. Then $\left(x_{i}+X_{i}\right) \cap Q=\emptyset$. Take $x=\left(x_{0}, \ldots, x_{k-1}\right)$. Then $(x+X) \cap \bigcup_{m<k} \mathbb{R}^{m} \times Q \times$ $\mathbb{R}^{k-m-1}=\emptyset$.

Assume that $X \in \mathcal{H} \cap \mathrm{SMZ} \cap P\left(\mathbb{R}^{k}\right)$ and $Y \in \mathrm{SMZ} \cap P\left(\mathbb{R}^{l}\right)$. It is well known (and easy to see) that $\mathcal{H}$ and SMZ satisfy the assumptions of Lemma 3.6. Take

$$
Q=\bigcup_{m, n \in \mathbb{Z}}\left(m \cdot \mathbb{Q}_{2}+n\right) .
$$

Then by Lemma 3.6, we may assume that $X$ is disjoint from $\bigcup_{m<k} \mathbb{R}^{m} \times Q \times \mathbb{R}^{k-m-1}$, and $Y$ is disjoint from $\bigcup_{m<l} \mathbb{R}^{m} \times Q \times \mathbb{R}^{l-m-1}$.

For each $n$, set $X_{n}=X \cap[-n, n]^{k}$ and $Y_{n}=Y \cap[-n, n]^{l}$. Then $X_{n}$ is a closed subset of $X$ and therefore has the Hurewicz property. Moreover, $X_{n}$ and $Y_{n}$ have strong measure zero, and $X \times Y=\bigcup_{n} X_{n} \times Y_{n}$. Since SMZ is preserved under countable unions, it is enough to show that $X_{n} \times Y_{n}$ has strong measure zero for each $n$. Transforming (each coordinate of) $X_{n}, Y_{n}$ with the bi-Lipschitz homeomorphism $x \mapsto(n+x) / 2 n$, we may assume (by our choice of $Q$ !) that $X_{n}, Y_{n} \subseteq\left([0,1] \backslash \mathbb{Q}_{2}\right)^{k}$.

So assume that $X \in \mathcal{H} \cap \mathrm{SMZ} \cap P\left(\left([0,1] \backslash \mathbb{Q}_{2}\right)^{k}\right)$, and $Y \in \mathrm{SMZ} \cap P\left(\left([0,1] \backslash \mathbb{Q}_{2}\right)^{l}\right)$ Since $T^{k}: \mathcal{C}^{k} \rightarrow\left([0,1] \backslash \mathbb{Q}_{2}\right)^{k}$ is a homeomorphism, $T^{-k}[X]$ has the Hurewicz property. By Proposition 3.3, $T^{-k}[X], T^{-l}[Y] \subseteq \mathcal{C}$ have strong measure zero. By Scheepers' 
Theorem in $\mathcal{C}, T^{-k}[X] \times T^{-l}[Y]$ has strong measure zero. As $T^{k} \times T^{l}$ is uniformly continuous, $X \times Y$ has strong measure zero.

\section{Borel's Conjecture And CONJUnCtion of PROPERTIES}

To put things in a wider context, we briefly describe the general framework. Let $X$ be a topological space. An open cover $\mathcal{U}$ of $X$ is an $\omega$-cover of $X$ if $X$ is not in $\mathcal{U}$ and for each finite subset $F$ of $X$, there is a set $U \in \mathcal{U}$ such that $F \subseteq U$. $\mathcal{U}$ is a $\gamma$-cover of $X$ if it is infinite and for each $x$ in $X, x \in U$ for all but finitely many $U \in \mathcal{U}$. Let $\mathcal{O}, \Omega$, and $\Gamma$ denote the collections of all countable open covers, $\omega$-covers, and $\gamma$-covers of $X$, respectively. Let $\mathfrak{U}$ and $\mathfrak{V}$ be collections of covers of a space $X$. Following are selection hypotheses which $X$ might satisfy or not satisfy.

$\mathrm{S}_{1}(\mathscr{A}, \mathscr{B})$ : For each sequence $\left\{\mathcal{U}_{n}\right\}_{n \in \mathbb{N}}$ of members of $\mathscr{A}$, there exist members $U_{n} \in \mathcal{U}_{n}$, $n \in \mathbb{N}$, such that $\left\{U_{n}\right\}_{n \in \mathbb{N}} \in \mathscr{B}$.

$\mathrm{S}_{\text {fin }}(\mathscr{A}, \mathscr{B})$ : For each sequence $\left\{\mathcal{U}_{n}\right\}_{n \in \mathbb{N}}$ of members of $\mathscr{A}$, there exist finite (possibly empty) subsets $\mathcal{F}_{n} \subseteq \mathcal{U}_{n}, n \in \mathbb{N}$, such that $\bigcup_{n \in \mathbb{N}} \mathcal{F}_{n} \in \mathscr{B}$.

$\mathrm{U}_{\text {fin }}(\mathscr{A}, \mathscr{B})$ : For each sequence $\left\{\mathcal{U}_{n}\right\}_{n \in \mathbb{N}}$ of members of $\mathscr{A}$ which do not contain a finite subcover, there exist finite (possibly empty) subsets $\mathcal{F}_{n} \subseteq \mathcal{U}_{n}, n \in \mathbb{N}$, such that $\left\{\cup \mathcal{F}_{n}\right\}_{n \in \mathbb{N}} \in \mathscr{B}$.

$\mathrm{U}_{\text {fin }}(\mathcal{O}, \Gamma)$ is the Hurewicz property, $\mathrm{S}_{f i n}(\mathcal{O}, \mathcal{O})$ is the Menger property, $\mathrm{S}_{1}(\mathcal{O}, \mathcal{O})$ is Rothberger's property $C^{\prime \prime}$, and $\mathrm{S}_{1}(\Omega, \Gamma)$ is the $\gamma$-property.

Many equivalences hold among these properties, and the surviving ones appear in Figure 1 (where an arrow denotes implication), to which no arrow can be added except perhaps from $\mathrm{U}_{f i n}(\mathcal{O}, \Gamma)$ or $\mathrm{U}_{\text {fin }}(\mathcal{O}, \Omega)$ to $\mathrm{S}_{f i n}(\Gamma, \Omega)[3]$.

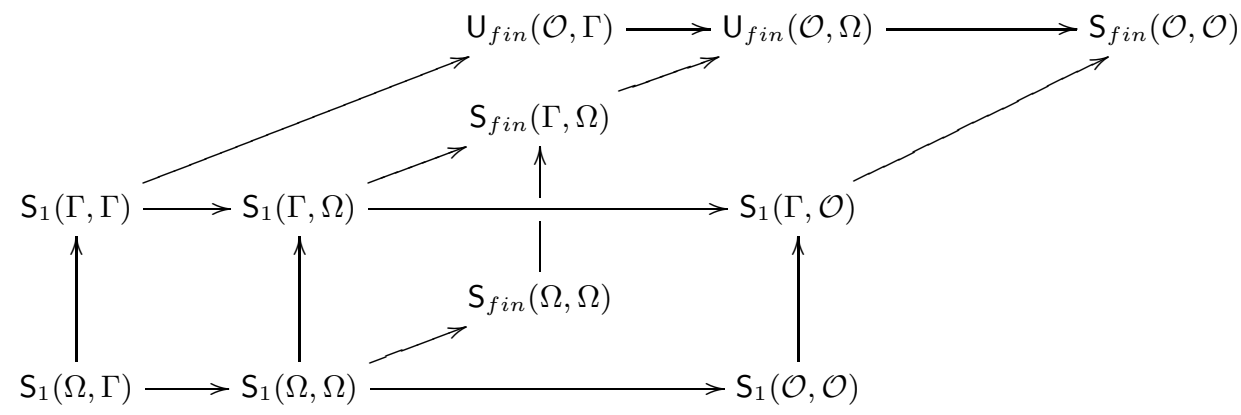

Figure 1. The Scheepers Diagram

Let us write $B C(P)$ for the Borel Conjecture for metric spaces with property $P$, that is, the hypothesis that every metric space with property $P$ is countable. Laver proved that $B C(\mathrm{SMZ})$ is consistent. Since $\mathrm{S}_{1}(\mathcal{O}, \mathcal{O})$ implies strong measure zero, it follows that $B C\left(\mathrm{~S}_{1}(\Omega, \Gamma)\right), B C\left(\mathrm{~S}_{1}(\Omega, \Omega)\right)$, and $B C\left(\mathrm{~S}_{1}(\mathcal{O}, \mathcal{O})\right)$ are all consistent. On the other hand, all other classes in the Scheepers Diagram provably contain uncountable sets of reals $[3,2]$, and therefore cannot satisfy $B C$.

In [6] Miller proves that $B C\left(\mathrm{~S}_{1}(\mathcal{O}, \mathcal{O})\right)$ implies $B C(\mathrm{SMZ})$, but $B C\left(\mathrm{~S}_{1}(\Omega, \Gamma)\right)$ does not imply $B C(\mathrm{SMZ})$. We will extend this result. With regards to the Scheepers 
Diagram 1, the best we can get is that $B C\left(\mathrm{~S}_{1}(\Omega, \Omega)\right)$ implies $B C(\mathrm{SMZ})$ (and therefore $B C\left(\mathrm{~S}_{1}(\Omega, \Gamma)\right)$ does not imply $\left.B C\left(\mathrm{~S}_{1}(\Omega, \Omega)\right)\right)$. We will prove a stronger result.

Definition 4.1. For a fixed topological space $X, \Omega^{g p}$ denotes the collection of open $\omega$-covers $\mathcal{U}$ of $X$ such that: There exists a partition $\mathcal{P}$ of $\mathcal{U}$ into finite sets such that for each finite $F \subseteq X$ and all but finitely many $\mathcal{F} \in \mathcal{P}$, there exists $U \in \mathcal{F}$ such that $F \subseteq U[4]$.

$\mathrm{S}_{1}\left(\Omega, \Omega^{g p}\right)$ is strictly stronger than $\mathrm{S}_{1}(\Omega, \Omega)[4]$.

Theorem 4.2. $B C\left(\mathrm{~S}_{1}\left(\Omega, \Omega^{g p}\right)\right)$ implies (and is therefore equivalent to) $B C(\mathrm{SMZ})$.

Proof. If $\aleph_{1}=\mathfrak{b}$ then by [2] there exists an uncountable set of reals $X$ satisfying $\mathrm{S}_{1}\left(\Omega, \Omega^{g p}\right)$.

Assume that $\aleph_{1}<\mathfrak{b}$, and $B C(\mathrm{SMZ})$ fails. Take any strong measure zero set $X$ with $|X|=\aleph_{1}$. Then $|X|<\mathfrak{c}$ and by a result of Carlson [1, Lemma 8.1.9], we may assume that $X \subseteq \mathbb{R}$. As $|X|<\mathfrak{b}$, all finite powers of $X$ have the Hurewicz property. By Scheepers' Theorem 1.10, $X^{2}=X \times X$ has strong measure zero, therefore $X^{3}=X \times X^{2}$ has strong measure zero, etc.

By [4], $\mathrm{S}_{1}\left(\Omega, \Omega^{g p}\right)$ is equivalent to all finite powers having strong measure zero and satisfying the Hurewicz property.

The arguments in the last proof actually establish the following.

Theorem 4.3. For a set of reals $X$, the following are equivalent:

(1) $X$ satisfies $\mathrm{S}_{f i n}\left(\Omega, \Omega^{g p}\right)$ and has strong measure-zero,

(2) $X$ satisfies $\mathrm{S}_{\text {fin }}\left(\Omega, \Omega^{g p}\right)$ and $\mathrm{S}_{1}(\mathcal{O}, \mathcal{O})$,

(3) $X$ satisfies $\mathrm{S}_{\text {fin }}\left(\Omega, \Omega^{g p}\right)$ and is meager-additive,

(4) $X$ satisfies $\mathrm{S}_{f i n}\left(\Omega, \Omega^{g p}\right)$ and $\mathrm{S}_{1}(\Omega, \Omega)$,

(5) $X$ satisfies $\mathrm{S}_{1}\left(\Omega, \Omega^{g p}\right)$.

Proof. Clearly, $5 \Rightarrow 4 \Rightarrow 2 \Rightarrow 1$, and $3 \Rightarrow 1$.

$(1 \Rightarrow 5)$ Assume that $(1)$ holds. In [4] it is proved that $\mathrm{S}_{f i n}\left(\Omega, \Omega^{g p}\right)$ is equivalent to satisfying the Hurewicz property $\mathrm{U}_{f i n}(\mathcal{O}, \Gamma)$ in all finite powers. By Scheepers' Theorem 1.10, all finite powers of $X$ satisfy $\bigcup_{f i n}(\mathcal{O}, \Gamma)$ and have strong measure zero. By [4], $X$ satisfies $\mathrm{S}_{1}\left(\Omega, \Omega^{g p}\right)$

$(1 \Rightarrow 3)$ In $[7]$ it is proved that every strong measure zero set of reals with the Hurewicz property is meager additive.

The theorem also holds when $X \subseteq \mathcal{C}$. In this case, the quoted assertion in the last proof can be proved directly - see Theorem A.3 in the appendix.

Acknowledgements. We thank Marcin Kysiak for his comment following Theorem 2.3, and Tomek Bartoszyński for the permission to include here his proof of Theorem A.2.

\section{Appendix A. Direct PRoOfs of QUOTED THEOREMS}

Following is Bartoszyński's (unpublished) combinatorial proof of Scheepers' Theorem 1.10 in $\mathcal{C}$. The proof uses the following characterization of strong measure zero. 
Lemma A.1 ([1, Lemma 8.1.13]). For $X \subseteq \mathcal{C}$, the following are equivalent:

(1) $X$ has strong measure zero,

(2) For each $f \in \mathbb{N}^{\mathbb{N}}$ there exists a function $g$ such that $g(n) \in\{0,1\}^{f(n)}$ for all $n$, and for each $x \in X$ there exist infinitely many $n$ such that $x\lceil f(n)=g(n)$,

(3) For each increasing sequence $\left\{m_{n}\right\}_{n \in \mathbb{N}}$ there exists $z \in \mathcal{C}$ such that for each $x \in X$ there exist infinitely many $n$ such that $x \uparrow\left[m_{n}, m_{n+1}\right)=z \uparrow\left[m_{n}, m_{n+1}\right)$.

Let $\mathbb{N} \nearrow^{\mathbb{N}}$ denote the subspace of the Baire space $\mathbb{N}^{\mathbb{N}}$ consisting of the increasing functions in $\mathbb{N}^{\mathbb{N}}$. In [14] it is proved that if $X$ has the Hurewicz property and $\Psi: X \rightarrow$ $\mathbb{N} \nearrow^{\mathbb{N}}$ is continuous, then $\Psi[X]$ admits some slalom $h \in \mathbb{N} \nearrow \mathbb{N}$, that is, such that for each $x \in X$ and all but finitely many $n$, there exists $k$ such that $h(n) \leq \Psi(x)(k)<h(n+1)$. This fact will be used in the proof.

Theorem A.2. Assume that $X \subseteq\{0,1\}^{\mathbb{N}}$ has the Hurewicz property and strong measure zero, and $Y \subseteq \mathcal{C}$ has strong measure zero. Then $X \times Y$ has strong measure zero.

Proof (Bartoszyński). Fix $f \in \mathbb{N}^{\nearrow \mathbb{N}}$ and let $g$ be as in Lemma A.1 for $X$ and $f$. Define a function $\Psi: X \rightarrow \mathbb{N}^{\nearrow \mathbb{N}}$ so that for each $x \in X, \Psi(x)$ is the increasing enumeration of the set $\{n: x\lceil f(n)=g(n)\}$. Then $\Psi$ is continuous, thus there exists $h \in \mathbb{N} \nearrow^{\mathbb{N}}$ such that for each $x \in X$ and all but finitely many $n$, there exists $k$ such that $h(n) \leq \Psi(x)(k)<h(n+1)$.

Consider a mapping $\Phi$ defined on $Y$ by

$$
\Phi(y)(n)=\langle(g(k), y\lceil f(k)): h(n) \leq k<h(n+1)\rangle .
$$

Then $\Phi$ is uniformly continuous. Thus (essentially, by Lemma A.1) there exists a function $r$ such that for all $y \in Y$ there exist infinitely many $n$ such that $\Phi(y)(n)=$ $r(n)$. From $r$ we decode a function $s$ such that $s(n) \in\{0,1\}^{f(n)} \times\{0,1\}^{f(n)}$ by $s(k)=r(n)(k)$ where $n$ is such that $h(n) \leq k<h(n+1)$.

Then for all $x \in X$ and $y \in Y$ there exist infinitely many $n$ such that $(x \uparrow f(n), y \uparrow$ $f(n))=s(n)$, which shows that $X \times Y$ has strong measure zero.

Using the bi-Lipschitz transformations $\Psi_{k}$ of Section 1, we obtain Scheepers' Theorem in $\tilde{P}(\mathcal{C})$ from Theorem A.2.

We can prove a result which is stronger (in light of the previous sections). Following is a direct, combinatorial proof of one of the main theorems in [7] when restricted to the Cantor space.

Theorem A.3 ([7]). Assume that $X \in \tilde{P}(\mathcal{C}), X$ has the Hurewicz property $\mathrm{U}_{\text {fin }}(\mathcal{O}, \Gamma)$, and strong measure zero. Then $X$ is meager-additive.

Proof. By Section 1, it suffices to prove the result for $X \subseteq \mathcal{C}$.

Assume that $\left\{m_{n}\right\}_{n \in \mathbb{N}}$ is an arbitrary increasing sequence. By Lemma 1.12, it suffices to find a sequence $\left\{l_{n}\right\}_{n \in \mathbb{N}}$ and $z \in \mathcal{C}$ such that for each $x \in X$ and all but finitely many $n, l_{n} \leq m_{k}<m_{k+1} \leq l_{n+1}$ and $x \uparrow\left[m_{k}, m_{k+1}\right)=z \uparrow\left[m_{k}, m_{k+1}\right)$ for some $k$. 
By Lemma A.1, there exists $z \in \mathcal{C}$ such that for each $x \in X$ there exist infinitely many $n$ such that $x \uparrow\left[m_{n}, m_{n+1}\right)=z\left\lceil\left[m_{n}, m_{n+1}\right)\right.$. Again, for each $x \in X$ let $\Psi(x)$ be the increasing enumeration of these $n \mathrm{~s}$, and use the fact that $X$ has the Hurewicz property to find a slalom $h \in \mathbb{N}^{\nearrow^{\mathbb{N}}}$ for $\Psi[X]$.

Take $l_{n}=m_{h(n)}$ for each $n$. Fix $x \in X$. Since $h$ is a slalom for $\Psi[X]$, for all but finitely many $n$ there exists $k$ such that for $i=\Psi(x)(k), h(n) \leq i<h(n+1)$. Then

$$
l_{n}=m_{h(n)} \leq m_{i}<m_{i+1} \leq m_{h(n+1)}=l_{n+1},
$$

and by the definition of $\Psi(x), x \uparrow\left[m_{i}, m_{i+1}\right)=z \uparrow\left[m_{i}, m_{i+1}\right)$.

\section{REFERENCES}

[1] T. Bartoszyński and H. Judah, Set Theory: On the structure of the real line, A. K. Peters, Massachusetts: 1995.

[2] T. Bartoszyński and B. Tsaban, Hereditary topological diagonalizations and the MengerHurewicz Conjectures, Proceedings of the American Mathematical Society, to appear. http://arxiv.org/abs/math.LO/0208224

[3] W. Just, A. W. Miller, M. Scheepers, and P. Szeptycki, Combinatorics of open covers II, Topology and Its Applications, 73 (1996), 241-266.

[4] Lj. D. R. Kočinac and M. Scheepers, Combinatorics of open covers (VII): Groupability, Fundamenta Mathematicae 179 (2003), 131-155.

[5] A.W. Miller, Special subsets of the real line, in: Handbook of Set Theoretic Topology (eds. K. Kunen and J.E. Vaughan), 201-233, North Holland, Amsterdam: 1984.

[6] A. W. Miller, The $\gamma$-Borel conjecture, Archive for Mathematical Logic, to appear. http://arxiv.org/abs/math.LO/0312308

[7] A. Nowik, M. Scheepers, and T. Weiss, The algebraic sum of sets of real numbers with strong measure zero sets, The Journal of Symbolic Logic 63 (1998), 301-324.

[8] J. Pawlikowski and I. Recław, Parametrized Cichon's diagram and small sets, Fundamenta Mathematicae 147 (1995), 135-155.

[9] F. Rothberger, Sur des families indenombrables de suites de nombres naturels, et les problémes concernant la proprieté C, Proceedings of the Cambridge Philosophical Society 37 (1941), 109126.

[10] M. Scheepers, Combinatorics of open covers I: Ramsey theory, Topology and its Applications 69 (1996), 31-62.

[11] M. Scheepers, Finite powers of strong measure zero sets, The Journal of Symbolic Logic 64 (1999), 1295-1306.

[12] M. Scheepers and B. Tsaban, The combinatorics of Borel covers, Topology and its Applications 121 (2002), 357-382.

[13] B. Tsaban, Selection principles and the minimal tower problem, Note di Matematica 22 (2003), $53-81$.

[14] B. Tsaban, The Hurewicz covering property and slaloms in the Baire space, Fundamenta Mathematicae 181 (2004), 273-280.

[15] B. Tsaban, Some new directions in infinite-combinatorial topology, to appear in: Set Theory (eds. J. Bagaria and S. Todorčevic). http://arxiv.org/abs/math.GN/0409069

[16] T. Weiss and B. Tsaban, Topological diagonalizations and Hausdorff dimension, Note di Matematica 22 (2003), 83-92. 
Department of Applied Mathematics and Computer Science, The Weizmann InstiTUte of Science, Rehovot 76100, IsRAel

E-mail address: boaz.tsaban@weizmann.ac.il

$U R L:$ http://www.cs.biu.ac.il/ ${ }^{\sim}$ tsaban

Institute of Mathematics, Akademia Podlaska 08-119 Siedlce, Poland

E-mail address: tomaszweiss@go2.pl 